\title{
Effect of the magnetic-field orientation on the modulation period of the critical current of ramp-type Josephson junctions
}

\author{
J.-K. Heinsohn, R. Dittmann, ${ }^{a}$ J. Rodríguez Contreras, E. Goldobin, A. M. Klushin, \\ and M. Siegel \\ Institut für Schichten und Grenzflächen, Forschungszentrum Jülich GmbH, 52425 Jülich, Germany \\ D. Hagedorn, R. Pöpel, R. Dolata, F.-Im. Buchholz, and J. Niemeyer \\ Physikalisch-Technische Bundesanstalt, Bundesallee 100, 38116 Braunschweig, Germany
}

(Received 1 May 2001; accepted for publication 3 August 2001)

\begin{abstract}
We have investigated the dependence of the critical current $I_{C}$ on the value and orientation of an externally applied magnetic field $H$ for interface-engineered $\mathrm{YBa}_{2} \mathrm{Cu}_{3} \mathrm{O}_{7-x}$ ramp-type Josephson junctions. The results are compared with measurements of $\mathrm{Nb}$ ramp-type junctions with a PdAu interlayer. The $I_{C}$ versus $H$ dependences are similar to Fraunhofer patterns and their modulation period changes several orders of magnitude with the orientation of the magnetic field. For both junction types, the dependence of the modulation period on the orientation of the magnetic field can be well described by the change of the relevant projection of the junction area and the influence of flux-focusing. Therefore the features of the $I_{C}(H)$ curves have to be attributed to the ramp geometry and not to specific properties of the superconducting material. (c) 2001 American Institute of Physics. [DOI: 10.1063/1.1406969]
\end{abstract}

\section{INTRODUCTION}

For high temperature superconductor (HTS) electronics, ramp-type Josephson junctions belong to the most widely used type of Josephson junctions. Since for applications, where only a small number of Josephson junctions is needed, grain boundary junctions (GBJs) still play an important role; for more complex electronic circuits, ramp-type junctions are the most promising alternative. ${ }^{1}$

For low temperature superconductor (LTS) electronics, planar tunnel junctions are well established and already allow for the fabrication of complex electronic circuits. In order to increase the density of integration in electronic circuits, ramp-type junctions with normal conducting interlayers, which have already been fabricated in the early $1980 \mathrm{~s}^{2}{ }^{2}$ gain new interest because they are intrinsically shunted and possess small junction areas which can easily be scaled down to the submicron range. ${ }^{3}$

Due to the tilted junction area and the epitaxial film growth across step edges, the current distribution as well as the penetration and pinning of magnetic flux may be different from planar junction geometries and common for HTS and LTS ramp-type junctions. Therefore comparative investigations of these effects in LTS and HTS junctions are of general interest.

The dependence of the critical current $I_{C}$ of a Josephson junction on the applied magnetic field $H$ is a sensitive method of determining the distribution of the supercurrent in the junction. HTS ramp-type junctions with artificial epitaxial barrier layers often do not exhibit the typical Fraunhoferlike $I_{C}(H)$ dependence $\left[I_{C}(x) \propto|(\sin \pi x) / x|\right.$; where $x$ $\left.=\Phi / \Phi_{0}\right]$ which would be expected in the case of a homogeneous current distribution. This can be attributed to mi-

\footnotetext{
${ }^{\text {a)} E l e c t r o n i c ~ m a i l: ~ r . d i t t m a n @ f z-j u e l i c h . d e ~}$
}

croshorts in the barrier or to large spatial variations of the barrier properties. Within the last few years, a new technology of fabricating ramp-type junctions, called "interface engineered junctions" (IEJs) (Refs. 4-6, and references therein) has become a promising alternative to the technology of depositing a nonsuperconducting epitaxial barrier layer on the ramp edge. A treatment of the milled $\mathrm{YBa}_{2} \mathrm{Cu}_{3} \mathrm{O}_{7-x}$ (YBCO) ramp edge by milling with higher energies and a subsequent annealing step results in a barrier at the interface between the two superconducting electrodes. The improved homogeneity of the barrier results in $I_{C}(H)$ modulations up to $100 \%$ with $I_{C}(H)$ patterns quite similar to the Fraunhofer pattern. It has been shown by analyzing the $I_{C}(H)$ patterns by means of a phase retrieval algorithm that a homogeneous current distribution can be obtained in IEJs. ${ }^{7}$

Even if the $I_{C}(H)$ curves of the IEJs are not ideal Fraunhofer patterns, their shapes are quite regular and allow for the determination of the modulation periods and thereby the evaluation of effective magnetic fields and effective junction areas. The investigation of the modulation period for different orientations of the magnetic field is interesting because of the special geometry of ramp-type junctions. Whereas in planar junctions or bicrystal GBJs the superconducting electrodes, the barrier layer, and the current flow are oriented along the principal axes, and ramp-type junctions have a higher level of geometrical complexity. Investigations of the $I_{C}(H)$ curves for different orientations of the magnetic field give insight into the different relevant projections of the junction area and different local magnetic fields.

In Refs. 8 and 9 , the $I_{C}$-modulation periods of HTS ramp-type junctions were investigated for certain orientations of the magnetic field. The modulation period for two different orientations has been calculated by taking into account the influence of flux focusing. In this article we present investigations of the modulation period of $I_{C}$ in dependence 


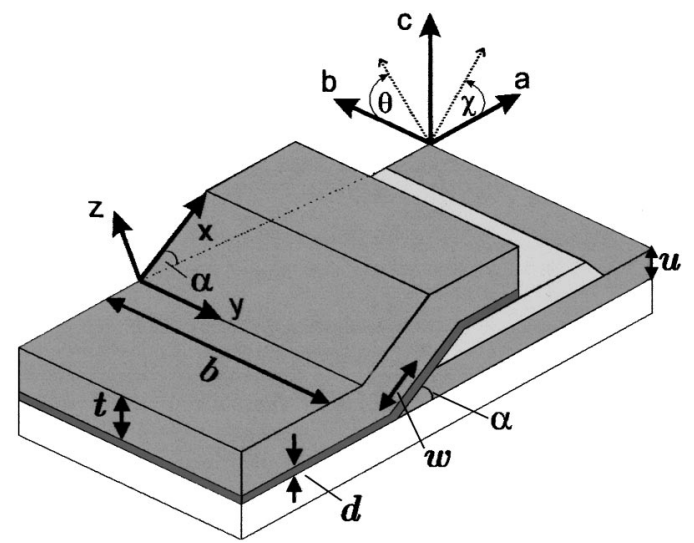

FIG. 1. Sketch of a ramp-type junction with the characteristic geometrical quantities that are important to describe the dependence of the $I_{C}$ modulation period on the orientation of the magnetic field. The $(a, b, c)$ coordinate system is relative to the crystalline axis of YBCO. It shows the two field orientations with the respective angles $\theta$ and $\chi$. The tilted coordinate system $(x, y, z)$ is relative to the ramp surface.

on the orientation angle for all reasonable orientations of the magnetic field. We will compare our measurements to calculations for the whole range of measured angles and draw conclusions to the influence of flux focusing and the penetration of magnetic fields in the ramp-type junctions.

When dealing with HTS ramp-type junctions, possibly some specific HTS properties have to be taken into account. These are the anisotropy of the unit cell and characteristic quantities like coherence length and penetration depth and the $d$-wave symmetry of the order parameter. Technological difficulties in HTS materials arise, for example, from the irregular, faceted, epitaxial film growth over the ramp. For this reason we compare the measurements on our IEJs with measurements on LTS ramp-type Josephson junctions with a $\mathrm{PdAu}$ interlayer in order to investigate especially the influence of the ramp geometry on the $I_{C}(H)$ modulation.

This article is structured as follows: After a brief description of the preparation processes and the typical properties of our junctions and the measurement setup in the next section, the $I_{C}(H)$ curves of the IEJs are discussed for various field orientations in Sec. III. In the following sections, the corresponding dependence of the modulation periods on the orientation of the magnetic field are discussed and explained by a theoretical model. Section VI summarizes the results.

\section{EXPERIMENTAL DETAILS}

\section{A. Junction preparation and properties}

In this section the preparation process and typical properties of the IEJs will be briefly described. A detailed description of the preparation process with a study of the significant process parameters can be found in Ref. 5. Figure 1 shows a sketch of a ramp-type junction in which the relevant geometrical parameters are noted.

First, a bilayer consisting of a $120 \mathrm{~nm}$ thick $\mathrm{YBa}_{1.95} \mathrm{La}_{0.05} \mathrm{Cu}_{3} \mathrm{O}_{7}$ film and a $220 \mathrm{~nm}$ thick $\mathrm{SrTiO}_{3}$ film is deposited on a $\mathrm{SrTiO}_{3}$ or $\mathrm{LaAlO}_{3}$ substrate in situ by pulsed laser deposition. The ramps are fabricated by conventional
TABLE I. Parameters of the junctions.

\begin{tabular}{lll}
\hline \hline Parameter & HTS junction & LTS junction \\
\hline Thickness $u$ of the base electrode & $120 \mathrm{~nm}$ & $80 \mathrm{~nm}$ \\
Thickness $t$ of the counterelectrode & $150 \mathrm{~nm}$ & $100 \mathrm{~nm}$ \\
Thickness $d$ of the barrier layer & 1 to $2 \mathrm{~nm}$ & $40 \mathrm{~nm}$ \\
Length $w$ of the ramp & $240 \mathrm{~nm}$ & $250 \mathrm{~nm}$ \\
Ramp angle $\alpha$ & $30^{\circ}$ & $19^{\circ}$ \\
Junction width $b$ & $3 \mu \mathrm{m}$ & $1.3 \mu \mathrm{m}$ \\
Flux-focusing factor $f$ & 24 & 15.6 \\
Critical temperature $T_{C}$ & $74 \mathrm{~K}$ & $7 \mathrm{~K}$ \\
London penetration depth & $\lambda_{L, a b} \approx 150 \mathrm{~nm} ;$ & $\lambda_{L} \approx 80 \mathrm{~nm}$ \\
& $\lambda_{L, c} \approx 750 \mathrm{~nm}$ & \\
Measurement temperature & $65 \mathrm{~K}$ & $5 \mathrm{~K}$ \\
Critical current density $j_{C}$ & $1.9 \times 10^{4} \mathrm{~A} / \mathrm{cm}^{2}$ & $2.4 \times 10^{5} \mathrm{~A} / \mathrm{cm}^{2}$ \\
Josephson penetration depth $\lambda_{J}$ & $2.12 \mu \mathrm{m}$ & $0.83 \mu \mathrm{m}$ \\
\hline \hline
\end{tabular}

photolithography and Ar-ion-beam milling. The interface engineering consists of a subsequent 3 min Ar-ion-milling procedure with increased energy followed by a $30 \mathrm{~min}$ annealing step in the deposition chamber. After the annealing procedure, the counterelectrode is deposited, and the junctions are patterned by conventional photolithography and Arion-beam milling. Finally, a $200 \mathrm{~nm}$ thick gold layer is evaporated and patterned by a lift-off process to provide electrical contacts. The relevant parameters of the IEJ studied in this article are summarized in Table I.

We believe that the interface treatment leads to the formation of a YBCO interface layer where strong cation disorder is present, which can be described by a series connection of a superconductor with reduced critical temperature and an insulator containing microshorts (constrictions) and localized states. ${ }^{6}$ The use of La-doped YBCO leads to junctions with increased normal resistances and decreased critical current densities which are in the short junction limit $\left(b / \lambda_{J}<4\right.$; with $\lambda_{J}=\sqrt{\hbar / 2 e \mu_{0} d^{\prime} j_{C}}$ and $d^{\prime}=d+2 \lambda_{L}$ ) for temperatures between 77 and $50 \mathrm{~K}^{6}$

The fabrication of the $\mathrm{Nb}$ ramp-type junctions with a $\mathrm{PdAu}$ interlayer is described in detail in Ref. 3. Briefly, in a first step, a $30 \mathrm{~nm} \mathrm{Al}_{2} \mathrm{O}_{3}$ layer is sputtered on the whole wafer subsequently serving as an etch stop. An $80 \mathrm{~nm}$ thick $\mathrm{Nb}$ layer is deposited by dc-magnetron sputtering followed by an rf-sputtered $50 \mathrm{~nm}$ thick $\mathrm{SiO}_{2}$ layer. In the $\mathrm{Al}_{2} \mathrm{O}_{3} / \mathrm{Nb} / \mathrm{SiO}_{2}$ sandwich assembly, the base electrodes are patterned first by wet etching of the $\mathrm{SiO}_{2}$ isolation layer with an ammonium fluoride etch mixture. Thereby, the photoresist edges are underetched in the order of the length of the ramp to be formed. Subsequently, the $\mathrm{Nb}$ ramp is produced by reactive ion etching with $\mathrm{CF}_{4}$ and an admixture of $\mathrm{O}_{2}$ in order to cause a draw back of the resist during the etching of the ramp. Before sputtering of the $\mathrm{PdAu}-\mathrm{Nb}$ bilayer, the surface is sputter cleaned with Ar. The junctions are patterned by reactive ion etching of $\mathrm{Nb}$ and Ar-beam etching of PdAu. The relevant dimensions and parameters of the LTS junction studied in this article are listed in Table I as well.

\section{B. Junction characterization}

We investigated the $I_{C}(H)$ curves of several hundred IEJs and choose two chips with typical junctions to measure 
the dependence of the $I_{C}(H)$ curves of seven junctions on the orientation in detail. Though the $I_{C}(H)$ curves of our IEJs often do not exhibit the symmetry and the clear second maxima of the junction presented in this article, a clear central maximum with an almost total suppression of the critical current in the minima is observed for the majority of the junctions. For this reason, the investigations of the modulation period, which are presented in the next section, are representative for all our IEJs.

The LTS ramp-type junctions have been characterized using a large number of samples. This has been published elsewhere. ${ }^{3,10}$ We investigated the $I_{C}(H)$ curves of one junction in dependence of the orientation in detail. Since the junction can be fabricated in a very reproducible way, the results presented in this article are typical for all low $T_{C}$ samples fabricated in the PTB.

The samples were measured in a He-flow cryostat. Temperatures between room temperature and $4 \mathrm{~K}$ could be adjusted by varying the He flow. For the HTS junctions, which were measured at $65 \pm 0.5 \mathrm{~K}$, the temperature fluctuations had no influence on their critical current. For the LTS junctions, which were measured at $5 \mathrm{~K}$, temperature changes of about $0.1 \mathrm{~K}$ already influenced the critical current significantly. Therefore the exact measurement temperature had to be recorded during the measurement of the LTS junctions.

The measurement temperatures were chosen so that the short-junction limit was valid. In this case, the $I_{C}(H)$ curves are expected to be Fraunhofer-like. ${ }^{11}$

The $I_{C}(H)$ curves are measured automatically. The $I_{C}$ is determined by sweeping the $I-V$ curve until a certain voltage criterion is obtained. In the case of the LTS ramp junctions, which have a very low normal resistance of $0.03 \Omega$, a $1 \mu \mathrm{V}$ criterion yields $I_{C}=33 \mu \mathrm{A}$ even at total suppression of the critical current by the external magnetic field.

The cryostat was located in the center of a Helmholtz coil. The magnetic field could be varied from -25 to $25 \mathrm{mT}$. It was adjusted automatically by a programmable current source which was controlled by a computer.

The orientation of the magnetic field relative to the sample could be adjusted by rotating the probe in the magnetic field of the Helmholtz coil. The samples were measured in two steps. First, the samples were mounted with their $a$ axis parallel to the rotatable axis of the probe and measurements with field orientations parallel to the $b$ axis were performed. In the second step, the samples were mounted with their $b$ axis parallel to the axis of the probe, and field orientations parallel to the $a$ axis were performed.

The relative position of the probe to the magnetic field can be adjusted within a certainty of $1^{\circ}$. The accuracy of the absolute angle between ramp and magnetic field also depends on the misadjustments of the sample on the chip carrier, of the probe in the cryostat, or of the junctions on the substrate during the microstructuring. Therefore the uncertainty of the absolute angle can be estimated to be $5^{\circ}-8^{\circ}$.

In the following sections we shall describe the dependence of the $I_{C}(H)$ modulation period on the orientation of the applied magnetic field $\mathbf{H}$. We discriminate between the orientation of $\mathbf{H}$ in the $b c$ plane (in the following called "perpendicular orientation") and the orientation in the $a c$ (a)

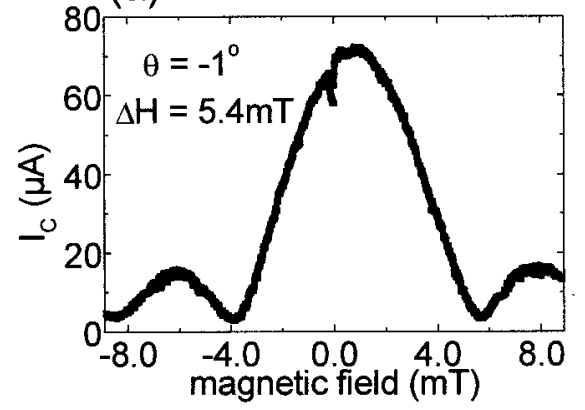

(b)

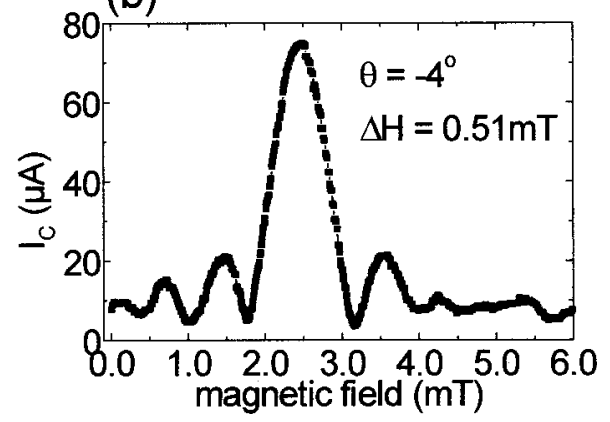

(c)

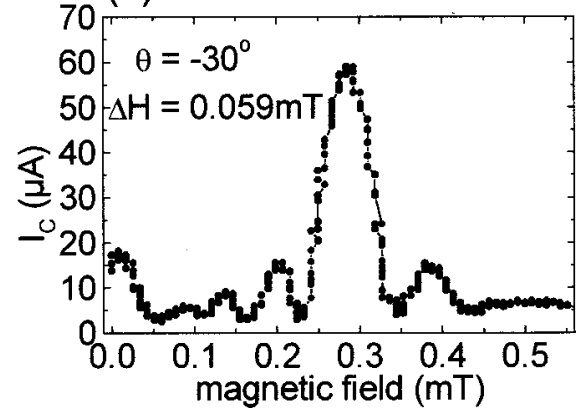

FIG. 2. $I_{C}(H)$ curves of the HTS junction at $65 \mathrm{~K}$ in perpendicular orientation at different angles $\theta$ of the magnetic field: (a) $-1^{\circ}$, (b) $-4^{\circ}$, and (c) $-30^{\circ}$.

plane ("parallel orientation"). In the perpendicular orientation the magnetic field is always perpendicular to the current flow. In the parallel orientation the magnetic field has a component parallel to the current flow. The case $\chi=90^{\circ}$ in the parallel orientation is identical to the case $\theta=90^{\circ}$ in the perpendicular orientation (see the coordinate system in Fig. 1).

\section{DEPENDENCE OF THE CRITICAL CURRENT ON THE MAGNETIC FIELD OF RAMP-TYPE JUNCTIONS}

\section{A. HTS-ramp-type junctions}

Figure 2 shows a series of $I_{C}(H)$ curves for different angles $\theta$ of the magnetic field in perpendicular orientation at $T \approx 65 \mathrm{~K}$. The shapes of the curves are quite similar to the Fraunhofer pattern, but the central maximum generally does not appear at zero magnetic field. This shift of the maximum is caused by trapped magnetic flux. Since the offset magnetic field does not exhibit a systematic distribution we conclude that the vortices are neither trapped predominantly at one special position in the junction, nor is a fixed number of 
(a)

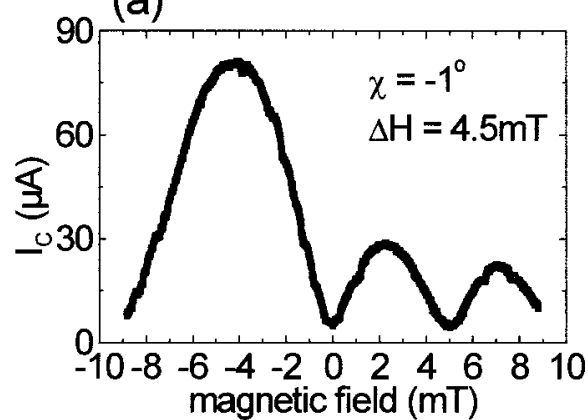

(b)
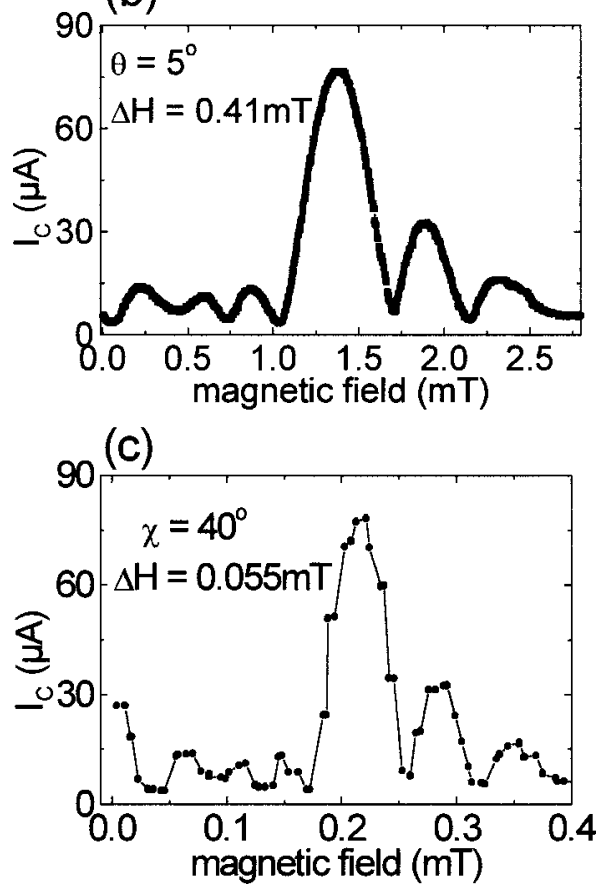

FIG. 3. $I_{C}(H)$ curves of the HTS junction at $65 \mathrm{~K}$ in parallel orientation at different angles $\chi$ of the magnetic field: (a) $-1^{\circ}$, (b) $5^{\circ}$, and (c) $40^{\circ}$.

vortices trapped in every measurement. Trapped vortices also lead to different maximum critical currents [e.g., $75 \mu \mathrm{A}$ in Fig. 2(b) and $60 \mu \mathrm{A}$ in Fig. 2(c)]. This is because a homogeneous external magnetic field cannot totally compensate the inhomogeneous field of trapped Abrikosov vortices.

The modulation period is determined by taking the difference between the magnetic fields for the central maximum and the first minimum. Differences between higher-order minima were not taken because for field orientations yielding the largest modulation periods the maximum magnetic field sometimes was not sufficient to suppress the critical current twice or more, especially when the central maximum was strongly shifted due to trapped flux. Whether the minimum for the negative or the positive polarity of the field was taken, was decided individually depending on the similarity to the Fraunhofer pattern of the respective part of the curve.

The most important result of these measurements, which can be seen in Figs. 2(a)-2(c) and will be discussed in detail later, is that the modulation period of the $I_{C}(H)$ patterns changes about two orders of magnitude for the angles from $-1^{\circ}$ to $-30^{\circ}$.

Figure 3 shows a series of $I_{C}(H)$ curves for different (a)

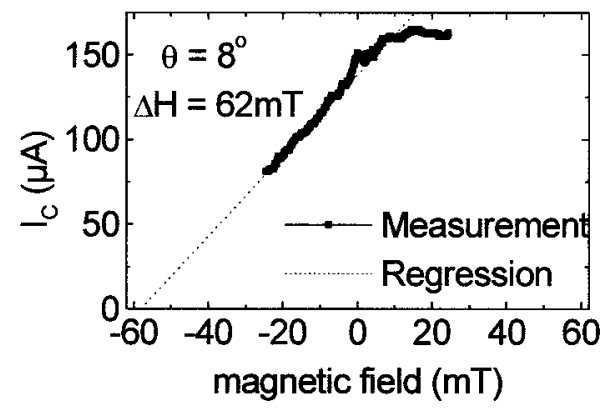

(b)

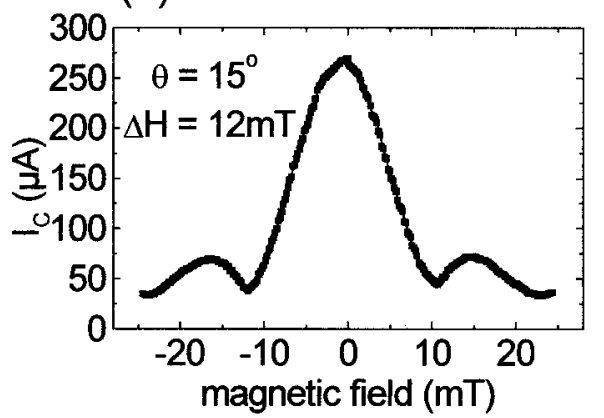

(c)

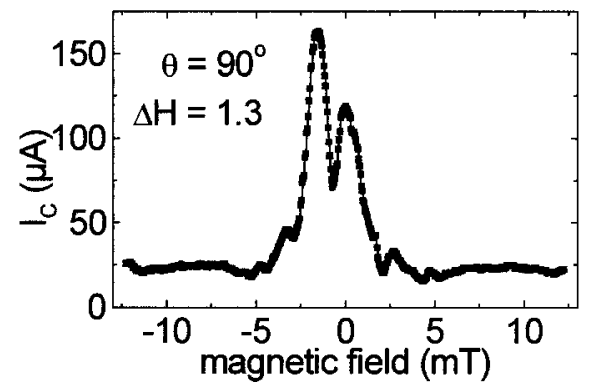

FIG. 4. $I_{C}(H)$ curves of the LTS junction at $T \approx 5 \mathrm{~K}$ in perpendicular orientation at different angles $\theta$ of the magnetic field: (a) $8^{\circ}$, (b) $15^{\circ}$, and (c) $90^{\circ}$.

angles $\chi$ of the magnetic field in parallel orientation at $T$ $\approx 65 \mathrm{~K}$. Again, the central maxima of the curves are shifted due to trapped magnetic flux. Trapped vortices are also responsible for the asymmetry of some curves as can be seen in Figs. 3(b) and 3(c), where the second maximum on the right-hand side is higher than the one on the left-hand side.

Similar to the observations in the case of the perpendicular orientation, the modulation period of the $I_{C}(H)$ curves changes about two orders of magnitude for $\chi$ between $-1^{\circ}$ and $40^{\circ}$ [see Figs. 3(a) $-3(\mathrm{c})$ ].

\section{B. LTS-ramp-type junctions}

Figure 4 shows a series of $I_{C}(H)$ curves for different angles $\theta$ of the magnetic field in perpendicular orientation at $T \approx 5 \mathrm{~K}$. Due to the limited available magnetic field, for the angles which yield the highest modulation periods the critical current could not even be totally suppressed once. In these cases, the first minimum was evaluated by a linear extrapolation of the $I_{C}(H)$ curve as depicted, e.g., in Fig. 4(a). The residual $I_{C}$ of $30 \mu \mathrm{A}$ in the minima of the curves in 


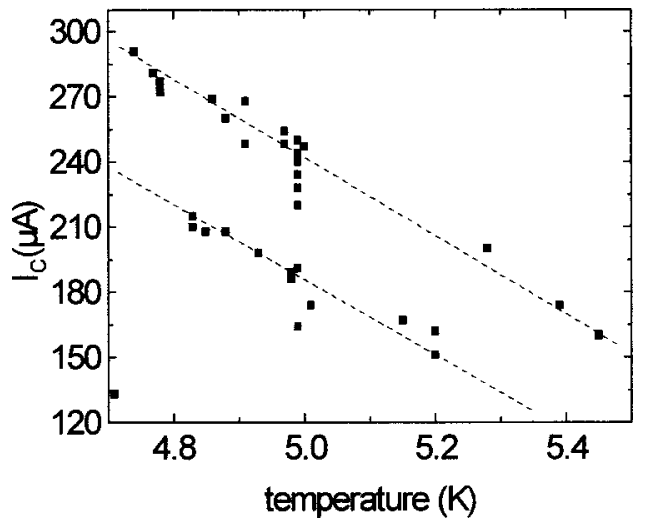

FIG. 5. Temperature dependence of the maximum critical current of the measurements of the LTS junction in perpendicular orientation. The dashed lines are a guide to the eye.

Figs. 4(b) and 4(c) originates from the $1 \mu \mathrm{V}$ criterion in the automated determination of the critical current as already mentioned in Sec. II B.

The common features of these $I_{C}(H)$ curves are the asymmetry of some curves [see Fig. 4(c)] and the shift of the maximum of the curve. Like in the HTS junctions, the shift of the maximum can be explained by trapped magnetic flux. Since trapped magnetic flux results also in a suppression of the maximum of the critical current we compared this value for the different measurements. Figure 5 shows the maximum critical current in dependence of the exact measurement temperature which varied between 4.7 and $5.5 \mathrm{~K}$. In this small temperature interval, one could expect the critical current to depend roughly linearly on the temperature. In Fig. 5 can be seen two distinct linear branches, which are sketched by the dashed lines, of which the lower branch corresponds to the asymmetric $I_{C}(H)$ curves. The occurrence of two branches suggests that the vortices are trapped predominantly at the same position. However, a systematic dependence of the possibility of flux trapping on the orientation angle of the magnetic field was not observed.

In Fig. 6 a series of $I_{C}(H)$ curves for different angles of the magnetic field $\chi$ in the parallel orientation at $5 \mathrm{~K}$ is shown. Again, the features discussed above are observed. Furthermore, for both orientations, the period of the $I_{C}(H)$ patterns changes about two orders of magnitude between $-90^{\circ}$ and $90^{\circ}$ [see Figs. 4(a)-4(c) and Figs. 6(a) $-6(\mathrm{c})$ ]. This effect, which was also observed for the HTS junctions, is analyzed in more detail in the next section.

\section{DEPENDENCE OF THE MODULATION PERIOD ON THE MAGNETIC FIELD ORIENTATION}

In this section the strong dependence of the modulation period on the angle of the magnetic field is analyzed for the two series of orientations. A theoretical model will be developed and compared with the experimental data obtained for the HTS and LTS samples. See Fig. 1 for the geometrical quantities used in this section. (a)

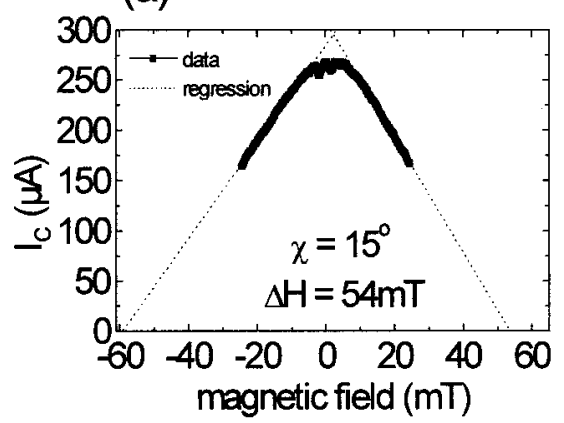

(b)

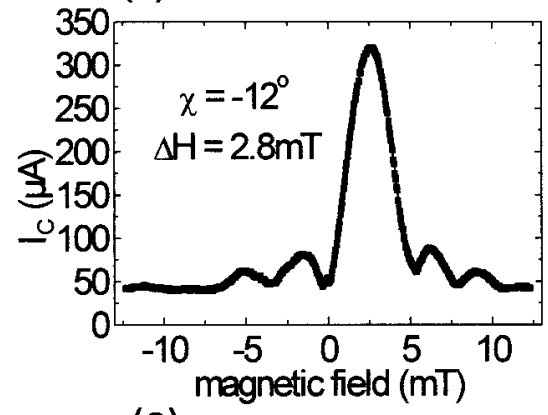

(c)

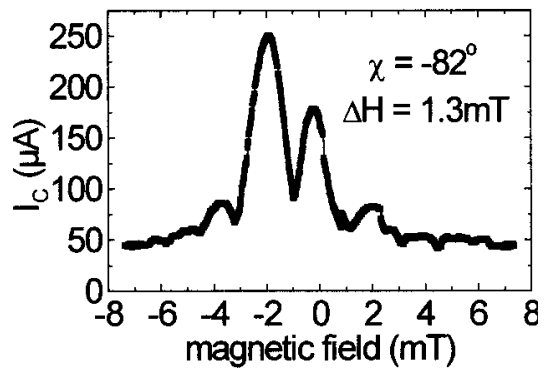

FIG. 6. $I_{C}(H)$ curves of the LTS junction $T \approx 5 \mathrm{~K}$ in parallel orientation at different angles $\chi$ of the magnetic field: (a) $15^{\circ}$, (b) $-12^{\circ}$, and (c) $-82^{\circ}$.

\section{A. Theoretical considerations}

For the evaluation of the modulation period it is useful to split the magnetic field into different components, $\mathbf{H}$ $=\left(H_{a}, H_{b}, H_{c}\right)$. This splitting makes sense due to the following reasons: First, the relevant projections of the junction area are different for the different field directions since $b$ is about an order of magnitude greater than $w$ (see Fig. 1). Second, due to the anisotropy of the YBCO crystal, the penetration depths $\lambda_{a b}$ and $\lambda_{c}$ are different in the HTS case. Third, the component $H_{c}$ of the magnetic field, which is perpendicular to the film, is enhanced due to the so-called flux focusing ${ }^{12}$ which can be understood in the following way.

A magnetic field which is applied perpendicular to a superconducting film of thickness $t$ is screened by the Meissner currents inside the film. If the film has a finite width, the field of the Meissner currents adds with the external field, thus resulting in an edge-field enhancement. This effect was calculated in Ref. 12 for a planar grain boundary junction. In the "thick-film limit," $b t>\lambda_{L}^{2}$, the demagnetization effects in the barrier region are modeled by an infinitely long ellipsoid of thickness $t$ and width $b$. This geometry leads to an 
enhancement of the magnetic field of the factor $f=1.2 \mathrm{~b} / \mathrm{t}$. The value of the flux-focusing factors $f$ calculated for the two types of junctions can be found in Table I. Since the effects of sharp edges and corners are not taken into account in these calculations, this formula is valid only within a factor of $3 .{ }^{12}$ The more complicated ramp geometry will probably lead to an even greater uncertainty. As there exist no calculations of flux-focusing effects for the ramp geometry, we use this formula, like other authors do., ${ }^{4,9}$ To account for the uncertainty of the flux-focusing-correction factor we introduce an additional correction factor $k: f^{\prime}=k f$. This leads to

$$
H_{c}^{\mathrm{eff}}=k f H \sin \theta .
$$

To analyze the modulation of critical current by the magnetic field, it is useful to introduce a second coordinate system $(x, y, z)$ which is depicted in Fig. 1. The $x$ axis is oriented parallel to the ramp, the $y$ axis is equal to the former $b$ axis, and the $z$ axis lies perpendicular to the ramp surface. The advantage of this coordinate system is that the $z$ component of $\mathbf{H}$ does not modulate the critical current because it is perpendicular to the junction area, and thus does not induce any flux-induced phase shift inside the junction.

In the perpendicular orientation, the vector of the magnetic field $\mathbf{H}$ is oriented parallel to the $b c$ plane $\left(H_{a}=0\right)$. The components of $\mathbf{H}$ which contribute to the modulation of $I_{C}$, taking into account Eq. (1), can be expressed in the following way:

$$
\begin{aligned}
& H_{x}=H_{c}^{\text {eff }} \sin \alpha=k f H \sin \theta \sin \alpha, \\
& H_{y}=H_{b}=H \cos \theta .
\end{aligned}
$$

A field $\mathbf{H}$ which has nonzero $x$ and $y$ components leads to a $I_{C}(H)$ dependence which is given by a product of two Fraunhofer patterns. ${ }^{11}$ In our case, the periods of the two Fraunhofer patterns are related to the flux components $H_{x}\left(2 \lambda_{x}+d\right) b$ and $H_{y}\left(2 \lambda_{y}+d\right) w$, respectively, where the parameters $\lambda_{x}$ and $\lambda_{y}$ denote the penetration depths when the magnetic field is applied in the $x$ direction or in the $y$ direction, respectively. The first minimum of the resulting Fraunhofer pattern is obtained at the value $\Delta H$ of the field at which one of the two flux components reaches $\Phi_{0}$. Taking into account Eqs. (2) and (3), we obtain

$$
\begin{aligned}
& \Delta H_{\perp}(\theta) \\
& \quad=\min \left(\frac{\Phi_{0}}{\left(2 \lambda_{x}+d\right) b|\sin \theta \sin \alpha| k f}, \frac{\Phi_{0}}{\left(2 \lambda_{y}+d\right) w|\cos \theta|}\right) .
\end{aligned}
$$

For $\mathrm{Nb}$ junctions, the penetration depth is a well-known parameter and is identical for the two directions. For YBCO junctions, the question is which values have to be taken for the penetration depths $\lambda_{x}$ and $\lambda_{y}$. For the field component $H_{x}$, the screening currents flow in the $b$ direction and $\lambda_{x}$ can be taken equal to $\lambda_{a b}$. For the field component $H_{y}$, screening currents flow along the ramp and have to flow partly in the $a b$ direction and partly in the $c$ direction. Therefore $\lambda_{y}$ is a superposition of the penetration depths in the two directions:

$$
\lambda_{y}=\sqrt{\lambda_{c}^{2} \sin ^{2} \alpha+\lambda_{a b}^{2} \cos ^{2} \alpha} .
$$

In the case of the parallel orientation, the vector of the magnetic field is oriented parallel to the $x z$ plane, $\mathbf{H}$ $=\left(H_{x}, 0, H_{z}\right)$. Since the $H_{z}$ component does not contribute to the modulation of the critical current, only the $x$ component of the flux has to be regarded. We express the $x$ component in terms of $H_{a}$ and $H_{c}$ to take into account flux focusing acting on $H_{c}$. Corresponding to Fig. 1 the following expressions are valid:

$$
\begin{aligned}
& H_{a}=H \cos \chi, \quad H_{c}^{\mathrm{eff}}=H \sin \chi k f, \\
& H_{x}=H_{a} \cos \alpha-H_{c}^{\mathrm{eff}} \sin \alpha \\
& =H \cos \chi \cos \alpha-k f H \sin \chi \sin \alpha .
\end{aligned}
$$

The resulting flux component for the $x$ direction is $H_{x}\left(2 \lambda_{x}+d\right) b$. Due to Eq. (6), the expression of the modulation period in the parallel orientation, $\Delta H_{\|}(\chi)$ is

$$
\Delta H_{\|}(\chi)=\frac{\Phi_{0}}{\left(2 \lambda_{x}+d\right) b|\cos \chi \cos \alpha-k f \sin \chi \sin \alpha|} .
$$

Without flux focussing, we would have a singularity at $\chi=90^{\circ}-\alpha$ because, as already mentioned above, a field perpendicular to the ramp area will not influence the critical current and therefore result in $\Delta H_{\|}=\infty$. Due to the flux focusing, the position of the singularity will be shifted towards $\chi=0$ depending on the flux focusing factors $k f$. This can be explained by the fact that flux focusing rotates the effective magnetic field relative to the external magnetic field direction.

\section{B. Comparison of the experimental data of the HTS junctions with the theoretical model}

The symbols in Fig. 7 show the experimentally measured dependences of the modulation period $\Delta H$ on the angles $\chi$ and $\theta$ of the magnetic field. For both parallel and perpendicular orientations there is a sharp maximum at $\chi$ $\approx 0$ and $\theta \approx 0$ : At larger angles, $\Delta H$ becomes two orders of magnitude lower than at $\chi \approx 0$ and $\theta \approx 0$.

Below we fit the experimental dependences $\Delta H(\chi)$ and $\Delta H(\theta)$ using Eq. (7) for parallel orientation and using Eq. (4) for perpendicular orientation. While fitting we choose a set of parameters to obtain the best fit for both parallel and perpendicular orientations simultaneously.

At first, with the flux focusing factor $f=1.2 \mathrm{~b} / \mathrm{t}=24$ and without any additional correction $(k=1)$, using the typical values for the penetration depths $\lambda_{a b}=150 \mathrm{~nm}$ and $\lambda_{c}$ $=750 \mathrm{~nm}$, we get the dashed curves shown in Figs. 7(a) and 7 (b). The flux focusing factor $f \approx 24$ is large enough to shift the maximum of the $\Delta H(\chi)$ dependence towards $\chi \approx 4^{\circ}$ in accordance with Eq. (7). This fits the peak position within the accuracy of the angle determination of our experimental setup, mentioned in Sec. II B. For the perpendicular orientation, according to our theory, the maximum is always at $\theta$ $=0$ and in agreement with our measurements. At the same time, the dashed curves do not fit the data very well, especially at larger angles. In fact, the calculated values of $\Delta H$ are about one order of magnitude higher than the experimental ones. 

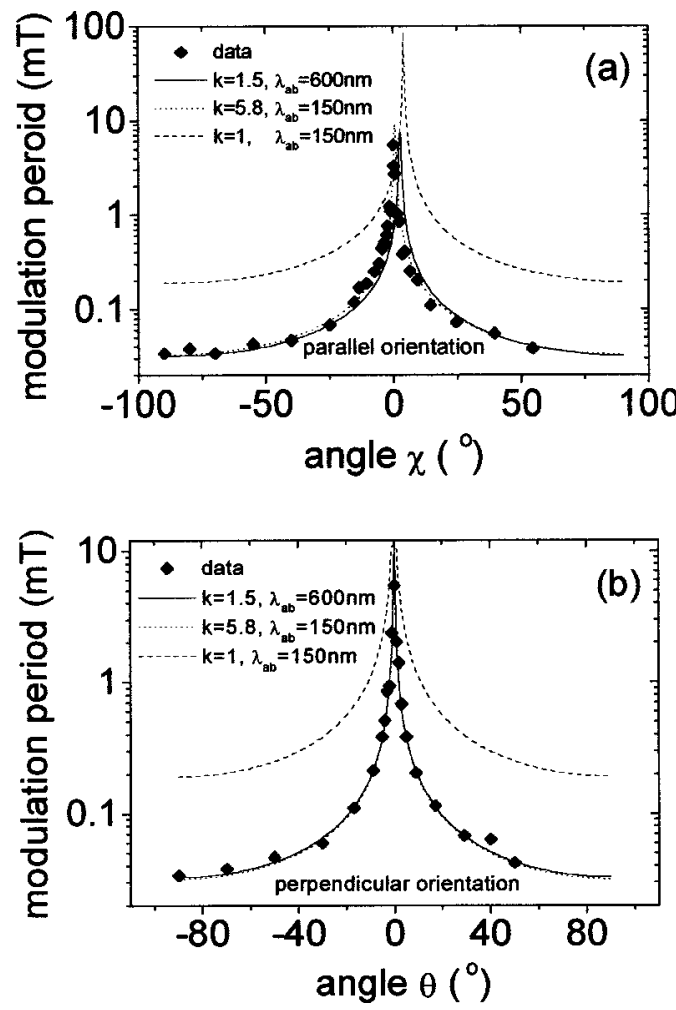

FIG. 7. (a) Angle dependence of the modulation period $\Delta H$ of the interfaceengineered HTS junction in perpendicular orientation. Together with the experimental data three theoretical curves according to Eq. (7) are plotted. Dotted line: $\lambda_{a b}=150 \mathrm{~nm}, k=1$. Dashed line: $\lambda_{a b}=150 \mathrm{~nm}, k=5.8$. Straight line: $\lambda_{a b}=600 \mathrm{~nm}, k=1.5$. (b) Angle dependence of the modulation period $\Delta H$ of the interface-engineered HTS junction in parallel orientation. Together with the experimental data three theoretical curves according to Eq. (4) are plotted. Dotted line: $\lambda_{a b}=150 \mathrm{~nm}, k=1$. Dashed line: $\lambda_{a b}$ $=150 \mathrm{~nm}, k=5.8$. Straight line: $\lambda_{a b}=600 \mathrm{~nm}, k=1.5$.

As a second approach we have chosen the flux-focusing correction factor $k$ as a free parameter and tried to get the best fitting of the experimental data. The best fitting takes place for $k=5.8$. The corresponding curves are shown in Figs. 7(a) and 7(b) as dotted lines. One can see that these curves fit the experimental data very well, but the value of the correction factor exceeds the uncertainty three times as stated in Ref. 12 and, therefore, $k=5.8$ cannot be considered as a reasonable value.

In a third approach we considered possible deviations from the parameters used in Eqs. (4) and (7) for the IEJs. One possible deviation is that the current does not flow homogeneously over the whole width $b$ of the junction. Gausepohl et al. ${ }^{8,9}$ introduced and calculated the "effective magnetic widths" of their junctions. But the reduced effective magnetic width of the junction would only further increase the modulation period and would explain our data even worse. On the other hand, the values for the London penetration depths can vary strongly because they depend on the structure of YBCO and on the temperature. Since the London penetration depth strongly increases near $T_{C}$, a layer with reduced $T_{C}$ at the interface would result in a $\lambda_{a b}$ which is actually higher than the $\lambda_{a b}$ in a usual undamaged YBCO. In the case of the IEJs, the existence of such an interface layer is reasonable because the ion-induced defects will gradually
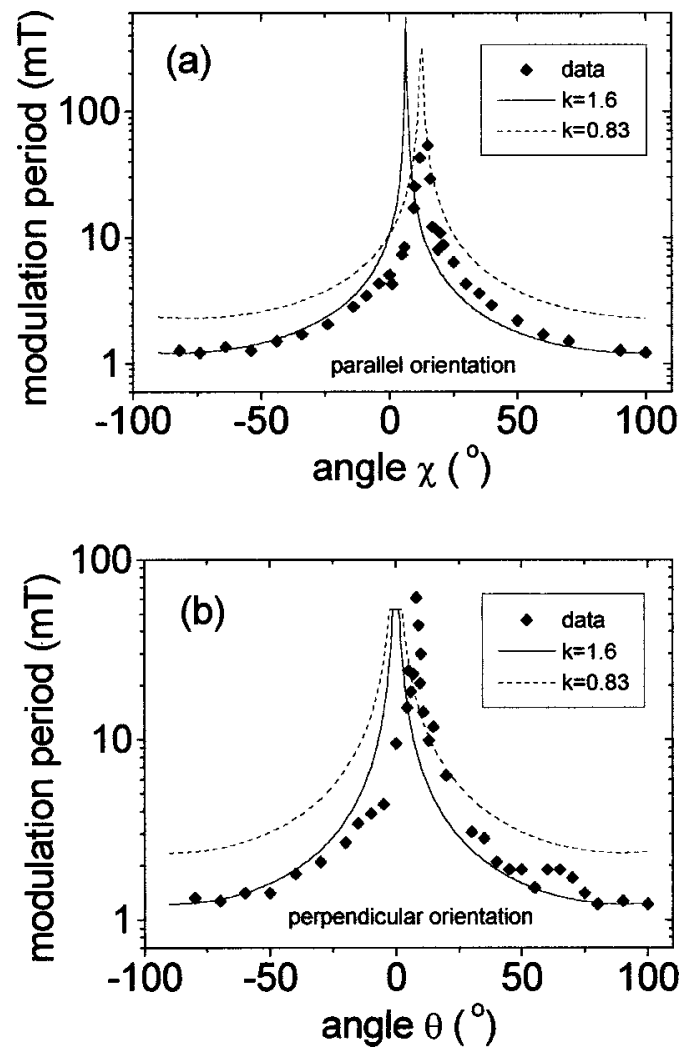

FIG. 8. (a) Angle dependence of the modulation period $\Delta H$ of the LTS junction in perpendicular orientation. Together with the experimental data three theoretical curves according to Eq. (7) are plotted. Dashed line: $k$ $=0.83$. Straight line: $k=1.6$. (b) Angle dependence of the modulation period $\Delta H$ of the LTS junction in parallel orientation. Together with the experimental data two theoretical curves according to Eq. (4) are plotted. Dashed line: $k=0.83$. Straight line: $k=1.6$.

decrease with the distance from the barrier. ${ }^{6}$ Therefore we use $\lambda_{a b}$ as a second fitting parameter and investigate its influence on the calculations. All other values $(b, w$, and $\alpha)$ in Eqs. (4) and (7) are determined with the accuracy of at least $30 \%$ and cannot explain the deviations. The solid lines in Figs. 7(a) and 7(b) show the calculations with $k=1.5$ and $\lambda_{a b}=600 \mathrm{~nm}$. The calculated curves fit the experimental data quite well for both parallel and perpendicular orientations.

\section{Comparison of the experimental data of the LTS junctions with the theoretical model}

The symbols in Figs. 8(a) and 8(b) show the experimentally measured dependences of the modulation period $\Delta H$ on the angles $\chi$ and $\theta$ for LTS Nb junctions. The dependences look very similar to the ones of HTS junctions [see Figs. 7(a) and $7(b)$ ]. For both orientations there is a sharp maximum at small angles and a reduction of the $\Delta H$ period by more than two orders of magnitude for larger angles.

For the $\mathrm{Nb}$ junctions, the London penetration depth is well known to be about $80 \mathrm{~nm}$. Therefore we use the value of the flux-focusing factor $f=15.6$ and the flux-focusing correction factor $k$ was used as the only fitting parameter. The different lines in Figs. 8(a) and $8(\mathrm{~b})$ show the $\Delta H(\chi)$ and $\Delta H(\theta)$ dependences calculated using Eqs. (7) and (4), respectively. Similar to the case of the HTS junctions we tried 
to fit both $\Delta H(\chi)$ and $\Delta H(\theta)$ dependences using the same value of the parameter $k$. The value of $k$ influences the value for $\Delta H$ for the large and for the parallel orientation, and additionally the position of the peak. For the perpendicular orientation, the height of the maximum of $\Delta H(\theta)$ does not depend on $k$ and fits the experimental data very well. Since the maximum of $\Delta H(\chi)$ is at $\chi=13^{\circ}$, we would need $k$ $=0.83$ to fit the position of the peak. The curves $\Delta H(\chi)$ and $\Delta H(\theta)$ calculated for this value of $k$ are shown by dashed lines in Fig. 8. On the other hand, for $k=0.83$ the value at high angles is about a factor of 2 higher than the experimental data. Since there is some error in the determination of the absolute angle we can allow some deviations of the peak position. Therefore we will take the value of $\Delta H$ at high angles as criterion. This gives the optimal value of $k=1.6$. The solid lines in Fig. 8 show the calculated $\Delta H(\chi)$ and $\Delta H(\theta)$ dependences for $k=1$.6. Since for the parallel orientation, $\Delta H(\chi)$ has a singularity at $\chi=\arctan [\cot (\alpha / k f)]$, the experimental values of $\Delta H(\chi)$ near the peak depend on the density of measurement points. Therefore it is not reasonable to discuss the deviation of the experimental data from the theoretical curve near the peak in Fig. 8(a). The maximum of the calculated curve for $k=1.6$ for the parallel orientation is at $\chi=7^{\circ}$ [see Fig. 8(a)]. The deviation of $6^{\circ}$ from the experimental peak at $13^{\circ}$ can be explained by the uncertainty of the experimental determination of $\chi$ as well as by the fact that $\theta$ may not be equal to zero precisely as well.

For the perpendicular orientation, the maximum of the experimental data is at $\theta=8^{\circ}$. This is according to the theoretical peak at $\theta=0^{\circ}$, about the same deviation of the position as in the case of the parallel orientation. Therefore it is reasonable to attribute this deviation to the systematic experimental error during the adjustment of the LTS sample as, for example, a misalignment of the sample in the sample holder.

\section{DISCUSSION}

The comparison of the dependences of the critical current on the value and orientation of the external magnetic field for HTS and LTS ramp-type junctions gives insight into the question of which deviations from the behavior of an ideal Josephson junction are due to the ramp geometry and which are due to specific material problems of HTS junctions.

In the IEJs as well in the LTS junctions, the $I_{C}(H)$ curves exhibit significant deviations from the Fraunhofer pattern. Where in IEJs this can at least be partly attributed to an inhomogeneous current distribution or HTS-specific effects, in LTS junctions only flux trapping can be the reason. In the case of YBCO ramp-type junctions flux trapping can be easily understood, since due to the difficult growth of the counterelectrode and the formation of facets during the heating prior to its deposition pinning centers are likely to be formed. This was shown in Ref. 13 by low-temperature scanning electron microscopy. However, this argument does not hold for LTS junctions since we can assume a homogeneous growth of the $\mathrm{Pd}-\mathrm{Au}$ interlayer and the $\mathrm{Nb}$ counterelectrode across the ramp. This leads to the conclusion that the ramp geometry itself is problematic with respect to flux trapping.

The observation of two distinct branches in the temperature dependence of the critical current shown in Fig. 5 suggests that vortices in LTS junctions are pinned predominantly at a certain position. Discrete branches of the $I_{C}(T)$ dependences were not observed for the IEJs. Nevertheless, more thorough investigations would have to be conducted in order to show whether the pinning centers are at random positions or at specific places due to the ramp geometry.

This general tendency of the ramp geometry to trap magnetic flux makes it difficult to use ramp-type junctions in electronic circuits. Therefore one has to think about using artificial pinning centers near the ramp area to prevent the flux from being pinned in the junction or using a superconducting groundplane to shield magnetic fields.

The dependence of the modulation period of the Fraunhofer patterns on the orientation of the magnetic field looks very similar for HTS and LTS junctions and can be described with the same model. Flux focusing strongly influences the modulation depth of both types of junctions. Due to the geometrical dimensions of the junctions, the field enhancement in $c$ direction due to flux focusing is stronger for the HTS junctions than for the LTS junctions. Since the flux-focusing correction factor $k$ which is needed to fit the data is similar for both junctions ( $k=1.5$ for HTS and $k=1.6$ for LTS) it can be concluded that this correction can mainly be attributed to the ramp junction geometry itself and not to specific field enhancement effects in the faceted HTS ramp-type junction.

The only significant difference between HTS and LTS junctions is that we have to assume $\lambda_{a b}=600 \mathrm{~nm}$ to receive a reasonable fit of the experimental data which is a strong deviation from its usual literature value of $150 \mathrm{~nm}$. In the case of the IEJs, this assumption can be justified by the existence of a layer of reduced $T_{c}$ near the interface because the ion induced defects will gradually decrease with the distance from the barrier. For the LTS junction, it can be assumed that the $\mathrm{Nb}$ film is not degraded near the $\mathrm{Pd}-\mathrm{Au}$ interface and no correction of the penetration depth is necessary.

\section{SUMMARY}

We investigated the $I_{C}(H)$ curves of interfaceengineered ramp-type junctions as well as LTS ramp-type junctions in dependence on the orientation of the magnetic field. Even if the shape of the $I_{C}(H)$ curves is very similar to the Fraunhofer pattern, the maximum critical current is always shifted to nonzero magnetic fields. This effect can be explained by trapped Abrikosov vortices. We have seen no difference in the probability of flux trapping for HTS and LTS junctions, so we conclude that the ramp geometry itself is prone to flux trapping.

For both types of junctions, the dependence of the modulation period of the $I_{C}(H)$ curves on the direction of the magnetic field has a sharp maximum at small angles where the magnetic field is oriented nearly parallel to the substrate plane. The modulation depth decreases about two orders of magnitude towards high angles where the magnetic field is perpendicular to the substrate plane. This dependence can be 
well described by the change of the relevant projection of the junction area and the influence of flux focusing. The main influence of flux focusing is a strong suppression of the modulation depth for magnetic fields which are nearly perpendicular to the substrate plane. The dependence of the modulation depth can be quantitatively fitted by the calculations if we introduce a flux-focusing-correction factor which accounts for the deviations from the infinitely long ellipsoid. This correction factor is nearly the same for HTS and LTS junctions and can therefore be mainly attributed to field enhancement in the ramp-junction geometry itself.

The only significant difference between HTS and LTS junctions is that we have to assume $\lambda_{a b}=600 \mathrm{~nm}$ to receive a reasonable fit of the experimental data which is a strong deviation from its usual literature value of $150 \mathrm{~nm}$. In the case of the IEJs, this assumption can be justified by the existence of an interface layer with reduced $T_{c}$.

However, most specifics of the $I_{C}$ vs $H$ patterns have to be attributed to the ramp geometry itself and not to specific properties of the superconducting material.

\section{ACKNOWLEDGMENTS}

We would like to thank R. Gross for helpful discussions. This work was supported by the Deutsche Forschungsge- meinschaft (Si 704/1-1, Ni 253/3-1) and the Bundesministerium für Bildung und Forschung (13N7534/1).

${ }^{1}$ M. Hidaka, T. Satoh, H. Terai, and S. Tahara, IEICE Trans. Electron. E80-C, 1226 (1997).

${ }^{2}$ R. F. Broom, A. Oosenbrug, and W. Walter, Appl. Phys. Lett. 37, 237 (1980).

${ }^{3}$ R. Pöpel, D. Hagedorn, T. Weimann, F.-Im. Buchholz, and J. Niemeyer, Supercond. Sci. Technol. 13, 148 (2000).

${ }^{4}$ B. H. Moeckly and K. Char, Appl. Phys. Lett. 71, 2526 (1997).

${ }^{5}$ J.-K. Heinsohn, R. H. Hadfield, and R. Dittmann, Physica C 326-327, 157 (1999).

${ }^{6}$ J.-K. Heinsohn, R. Dittmann, J. Rodríguez Contreras, J. Scherbel, A. Klushin, M. Siegel, C. L. Jia, S. Golubov, and M. Yu. Kupryanov, J. Appl. Phys. 89, 3852 (2001).

${ }^{7}$ M. Carmody, K. L. Merkle, Y. Huang, L. D. Marks, and B. H. Moeckly, Interface Sci. 8, 231 (2000).

${ }^{8}$ S. C. Gausepohl, M. Lee, and K. Char, Appl. Phys. Lett. 68, 2279 (1996).

${ }^{9}$ S. C. Gausepohl, M. Lee, S. J. Berkowitz, and W. H. Mallison, J. Appl. Phys. 82, 2427 (1997).

${ }^{10}$ R. Pöpel, D. Hagedorn, F.-Im. Buchholz, and J. Niemeyer, Inst. Phys. Conf. Ser. 167, 277 (2000).

${ }^{11}$ A. Barone and G. Paternó, Physics and Applications of the Josephson Effect (Wiley New York, 1982).

${ }^{12}$ P. A. Rosenthal, M. R. Beasely, K. Char, M. S. Colclough, and G. Zaharchuk, Appl. Phys. Lett. 59, 3482 (1991).

${ }^{13}$ A. Marx, K.-D. Husemann, B. Mayer, T. Nissel, R. Gross, M. A. J. Verhoeven, and G. J. Gerritsma, Appl. Phys. Lett. 64, 241 (1994). 